\title{
Performance Bounds of Multihop Wireless Communications with Blind Relays over Generalized Fading Channels
}

\author{
George K. Karagiannidis, Senior Member, IEEE
}

\begin{abstract}
In this letter, efficient performance bounds for multihop wireless communications systems with non-regenerative blind relays over non-identical Nakagami-n (Rice), Nakagami$m$ and Nakagami- $q$ (Hoyt) generalized fading channels, are presented. More specifically, the end-to-end signal-to-noise ratio (SNR) is formulated and upper bounded by using the wellknown inequality between harmonic and geometric mean of positive random variables. This bound is used to study important system's performance metrics: i) The moments of the end-to-end SNR which are obtained in closed-forms, and ii) The outage probability and the average error probability for coherent and non-coherent modulations, which are accurately approximated using the moments-based approach. Furthermore, new analytical formulae are derived for the gain of previously proposed semiblind relays in generalized fading environments. These kind of relays are used in numerical examples and computer simulations to verify the accuracy and to show the tightness of the proposed bounds.
\end{abstract}

Index Terms-Average SNR, average symbol error probability, moments, multihop communications, Nakagami- $q$ fading, Nakagami- $m$ fading, outage probability, Rice fading.

\section{INTRODUCTION}

$\mathbf{T}$ HE continuously rising demand for high data rate in current and future wireless networks has recently emerged multihop communications as a viable option for providing broader and more efficient coverage both in traditional (e.g. bent pipe satellites) and modern (e.g. ad-hoc, WLAN) communications networks. In contrast to conventional wireless networks, several intermediate terminals operates as relays between source and the destination in multihop systems. Recently, the concept of collaborative/cooperative diversity was proposed, to exploit the advantages of spatial diversity without the need of physical antenna arrays [1]-[7].

Looking through the up-to-date open technical literature, Hasna and Alouini studied the outage and the error performance of multihop systems with regenerative and nonregenerative relays over Rayleigh [1] and Nakagami- $m$ [2], [3] fading channels. The same authors, studied in [4] the performance of dual-hop systems equipped with non-regenerative blind relays (i.e. relays with fixed gain) and a specific fixed gain relay, called semi-blind, was proposed. Two other works related to the performance analysis of multihop transmissions with CSI-relays are included in [5] and [6]. Anghel and Kaveh

Manuscript received March 2, 2004; revised January 10, 2005; accepted March 24, 2005. The editor coordinating the review of this paper and approving it for publication is Prof. H. Yanikomeroglu. This paper was presented in part at IEEE Global Communications Conference (GLOBECOM'04), Dallas, Texas, November-December 2004.

G. K. Karagiannidis is with the Department of Electrical \& Computer Engineering, Aristotle University of Thessaloniki, GR-54124 Thessaloniki, Greece, (e-mail: geokarag@auth.gr).

Digital Object Identifier 10.1109/TWC.2006.03004 in [5] presented an exact average symbol error rate analysis for a cooperative network in a Rayleigh fading environment, while Boyer et al. in [6] presented an analysis for the physical layer of the multihop wireless communications channels. In the same work, was also introduced the concept of multihop diversity where each terminal receives signals form all the previous terminals along a single primary route. More recently, Laneman et al. developed and analyzed low-complexity cooperative diversity protocols that combat fading induced by multipath propagation in wireless networks [7]. In the same paper, were also developed performance characterizations in terms of outage events and associated outage probabilities, which measure robustness of the transmissions to fading in high SNRs. However, to the best of the author's knowledge there is no published work concerning the performance of multihop transmissions with blind relays over generalized fading channels, despite the fact that these kind of relays offer simplicity and ease of deployment, although they don't perform as well as the CSI-assisted relays [4].

The main contribution of this letter is the derivation of efficient bounds for the performance of multihop wireless communications systems with blind relays over independent but not necessarily identically distributed (i.d) Nakagami$n$ (Rice), Nakagami- $m$ and Nakagami- $q$ (Hoyt) generalized fading channels. A simple upper-bound is proposed for the end-to-end SNR by using the well-known inequality between harmonic and geometric mean of positive random variables (RVs). This bound is used to study important system's performance measures such as the moments of the end-to-end SNR which are expressed in closed-form as well as the outage and the average error probability for coherent (e.g. BPSK) and non-coherent (e.g. DPSK) modulation schemes, which are accurately approximated using the moments-based approach [8]. Furthermore, new analytical expressions are derived for the gain of previously proposed semi-blind relays in generalized fading environments. The analytical results are used in numerical and computer simulations examples to verify the accuracy of the presented mathematical analysis and to show the tightness of the proposed bounds.

The remainder of this letter is organized as follows. The multihop system under consideration is presented in the next section where a formula for the end-to-end SNR is derived and an upper-bound is proposed. In Section III, the moments of the end-to-end SNR are expressed in closed-form while in Section IV the end-to-end error and outage performance are studied. Finally, some concluding remarks are offered in Section V. 


\section{System And Channel Model}

An $N$-hop wireless communications system is considered, operating over independent but not necessarily i.d. fading channels. The source terminal $S$ communicates with the destination terminal $\mathrm{D}$ through the $N-1$ nodes-terminals, $T_{1}, T_{2}, \ldots, T_{N-1}$, which act as intermediate relays from one hop to the next.

\section{A. End-to-End SNR}

Assuming that terminal $\mathrm{S}$ is transmitting a signal $s(t)$ with an average power normalized to unity. Then, the end-to-end SNR at the output of terminal D can be written as [3, eq. (12)]

$$
\gamma_{\text {end }}=\frac{\prod_{i=1}^{N} \alpha_{i}^{2} G_{i-1}^{2}}{\sum_{i=1}^{N} N_{0, i}\left(\prod_{n=i+1}^{N} \alpha_{n}^{2} G_{n-1}^{2}\right)}
$$

where $\alpha_{i}$ is the fading amplitude of the $i$-th hop, $G_{i}$ is the gain of the $i$ th relay with $G_{0}=1$ and $N_{0, i}$ is the single-sided power spectral density of the additive white Gaussian noise (AWGN) at the input of $i$ th relay. When blind relays are used, they introduce fixed gain, $G_{i}$, given by

$$
G_{i}^{2}=\frac{1}{C_{i} N_{0, i}}, \quad i=1, \ldots, N-1
$$

where $C_{i}$ is a constant. Therefore, using (1) and (2), $\gamma_{\text {end }}$ can be written as

$$
\begin{aligned}
\gamma_{\text {end }} & =\left(\frac{1}{\gamma_{1}}+\frac{C_{1}}{\gamma_{1} \gamma_{2}}+\cdots+\frac{C_{1} C_{2} \cdots C_{N-1}}{\gamma_{1} \gamma_{2} \gamma_{3} \cdots \gamma_{N}}\right)^{-1} \\
& =\frac{1}{N}\left[\frac{1}{N} \sum_{n=1}^{N} \frac{1}{\beta_{n}}\right]^{-1}
\end{aligned}
$$

where

$$
\beta_{n}=\prod_{j=1}^{n} \frac{\gamma_{j}}{C_{j-1}}
$$

and $\gamma_{j}=\alpha_{j}^{2} / N_{0, j}$ is the instantaneous SNR of the $j$ th hop. It can be easily recognized that $\gamma_{\text {end }}$ is related to the harmonic mean, $\mathcal{H}_{N}^{\beta_{n}}$, of $\beta_{1}, \beta_{2}, \cdots, \beta_{N}$ with

$$
\gamma_{\text {end }}=\frac{1}{N} \mathcal{H}_{N}^{\beta_{n}}
$$

where by definition

$$
\mathcal{H}_{N}^{x_{i}} \triangleq\left(\frac{1}{N} \sum_{i=1}^{N} \frac{1}{x_{i}}\right)^{-1} .
$$

\section{B. An Upper Bound for the End-to-End SNR}

Unfortunately, due to the form of (3), it is difficult if not impossible - to derive closed-form expressions for several performance metrics of the multihop system under consideration. In order to overcome this problem, $\gamma_{e n d}$ is bounded using the well-known inequality between harmonic and geometric mean of positive RVs [9, Sec. 3.1]

$$
\mathcal{H}_{N}^{x_{i}} \leq \mathcal{G}_{N}^{x_{i}}
$$

where

$$
\mathcal{G}_{N}^{x_{i}} \triangleq\left(\prod_{i=1}^{N} x_{i}\right)^{\frac{1}{N}}
$$

being the geometric mean of $x_{i}$ s. The equality in (7) holds only for $x_{1}=x_{2}=\cdots=x_{N}$. Note, that the related inequality between arithmetic and geometric mean has been also used in [10] to determine a lower bound for the outage probability in cellular radio systems. Using (3), (5) and (7) and after manipulations an upper-bound for the end-to-end SNR, $\gamma_{b}$, is obtained as

$$
\gamma_{\text {end }} \leq \gamma_{b}=\mathcal{Z}_{N} \prod_{i=1}^{N} \gamma_{i}^{\frac{N+1-i}{N}}
$$

where $\mathcal{Z}_{N}$ is a constant given by

$$
\mathcal{Z}_{N}=\frac{1}{N} \prod_{i=1}^{N} C_{i}^{-\frac{N-i}{N}} .
$$

The form of $\gamma_{b}$ in (9) has the advantage of mathematical tractability over that in (3), while, as it will be shown in the next sections, it is a tight upper-bound for $\gamma_{e n d}$, which can be efficiently used to study the end-to-end system's performance.

\section{Semi-Blind Relays}

A subcategory of blind relays, which have previously proposed in [4] are the so-called semi-blind relays which consume the same average power with the corresponding CSI-assisted relays, i.e.

$$
G_{i, s b}^{2}=E\left\langle\frac{1}{\alpha_{i}^{2}+N_{0, i}}\right\rangle=\int_{0}^{\infty} \frac{f_{\alpha_{i}}\left(\alpha_{i}\right)}{\alpha_{i}^{2}+N_{0, i}} d \alpha_{i}
$$

with $f_{\alpha_{i}}\left(\alpha_{i}\right)$ being the probability density function (PDF) of the fading amplitude $\alpha_{i}$ and $E\langle\cdot\rangle$ denotes expectation. In [4], a formula for the gain of semi-blind relays was given when Rayleigh fading is assumed. Next, I present analytical expressions of this gain for generalized fading channels.

1) Nakagami-n (Rice) fading: Assuming that the multihop system operates in a Rice fading environment, then $\alpha_{i}$ follows a PDF given by [11]

$$
\begin{aligned}
f_{\alpha_{i}}\left(\alpha_{i}\right) & =\frac{2\left(K_{i}+1\right) \alpha_{i}}{\Omega_{i}} \exp \left[-\left(K_{i}+\frac{\left(K_{i}+1\right) \alpha_{i}^{2}}{\Omega_{i}}\right)\right] \\
& \times I_{0}\left(2 \sqrt{\frac{K_{i}\left(K_{i}+1\right)}{\Omega_{i}}} \alpha_{i}\right)
\end{aligned}
$$

where $K_{i}$ is the Rice factor at the $i$ th hop which ranges from 0 to $\infty, I_{0}(\cdot)$ is the zeroth-order modified Bessel function of the first kind and $\Omega_{i}$ is the signal's mean power, i.e. $\Omega_{i}=E\left\langle\alpha_{i}^{2}\right\rangle$. After using the infinite series representation for the $I_{0}(\cdot)[9$, eq. (9.6.10)] in (12) and changing the order of summation and integration (since the summand is Riemann integrable and 
converges uniformly on $[0, \infty))$, the gain, $G_{i, s b}$, can be written as

$$
\begin{aligned}
G_{i, s b}^{2} & =\frac{2\left(1+K_{i}\right) \exp \left(-K_{i}\right)}{\bar{\gamma}_{i} N_{0, i}} \sum_{n=0}^{\infty}\left[\frac{K^{n}\left(1+K_{i}\right)^{n}}{n ! \Gamma(n+1)\left(\bar{\gamma}_{i} N_{0, i}\right)^{n}}\right. \\
& \left.\times \int_{0}^{\infty} \frac{\alpha_{i}^{2 n+1} \exp \left[-\frac{\left(1+K_{i}\right) \alpha_{i}^{2}}{\bar{\gamma}_{i} N_{0, i}}\right]}{\alpha_{i}^{2}+N_{0, i}} d \alpha_{i}\right]
\end{aligned}
$$

where, $\bar{\gamma}_{i}=\Omega_{i} / N_{0, i}$, is the average SNR of the $i$ th hop. Therefore, using [12, eq. (3.353/5) and (8.352/3)] in (13) resulting to

$$
\begin{aligned}
G_{i, s b}^{2} & =\frac{\left(1+K_{i}\right) \exp \left[\frac{K_{i}+1}{\bar{\gamma}_{i}}-K_{i}\right]}{\bar{\gamma}_{i} N_{0, i}} \\
& \times \sum_{n=0}^{\infty}\left[\frac{K_{i}^{n}\left(1+K_{i}\right)^{n}}{n ! \bar{\gamma}_{i}^{n}} \Gamma\left(-n, \frac{K_{i}+1}{\bar{\gamma}_{i}}\right)\right]
\end{aligned}
$$

with $\Gamma(x, y)$ being the incomplete Gamma function defined in [12, eq. $(8.350 / 2)]$.

2) Nakagami-q (Hoyt) fading: Nakagami- $q$ (Hoyt) distribution is normally observed on satellite links subject to strong ionospheric scintillation and ranges from one-sided Gaussian to Rayleigh distribution [11]. When $\alpha_{i}$ follows Hoyt distribution, its PDF is given by [11]

$$
\begin{aligned}
f_{\alpha_{i}}\left(\alpha_{i}\right) & =\frac{\left(1+q_{i}^{2}\right) \alpha_{i}}{q_{i} \Omega_{i}} \exp \left[-\frac{\left(1+q_{i}^{2}\right)^{2} \alpha_{i}^{2}}{4 q_{i}^{2} \Omega_{i}}\right] \\
& \times I_{0}\left(\frac{\left(1-q_{i}^{4}\right) \alpha_{i}^{2}}{4 q^{2} \Omega_{i}}\right)
\end{aligned}
$$

where $0 \leq q_{i} \leq 1$ is the Hoyt fading parameter. Following the same procedure as above, the gain in (11) can be expressed as

$$
\begin{aligned}
G_{i, s b}^{2} & =\frac{\left(1+q_{i}^{2}\right)}{q_{i} \bar{\gamma}_{i} N_{0, i}} \sum_{n=0}^{\infty}\left[\frac{\left(1-q_{i}^{4}\right)^{2 n}}{2^{6 n} q_{i}^{4 n}\left(\bar{\gamma}_{i} N_{0, i}\right)^{2 n} n ! \Gamma(n+1)}\right. \\
& \left.\times \int_{0}^{\infty} \frac{\alpha_{i}^{4 n+1} \exp \left[-\frac{\left(1+q_{i}^{2}\right)^{2} \alpha_{i}^{2}}{4 q_{i}^{2} \bar{\gamma}_{i} N_{0, i}}\right]}{\alpha_{i}^{2}+N_{0, i}} d \alpha_{i}\right]
\end{aligned}
$$

which yields using [12, eq. (3.353/5) and (8.352/3)] to

$$
\begin{aligned}
G_{i, s b}^{2} & =\frac{\mu_{i}^{\frac{1}{2}}}{\bar{\gamma}_{i}^{\frac{1}{2}} N_{0, i}} \sum_{n=0}^{\infty}\left[\frac{\left(1-q_{i}^{4}\right)^{2 n} \exp \left(\mu_{i}\right)}{n ! 2^{6 n} q_{i}^{4 n} \bar{\gamma}_{i}^{2 n} \Gamma(n+1)}\right. \\
& \left.\times \Gamma(1+2 n) \Gamma\left(-2 n, \mu_{i}\right)\right]
\end{aligned}
$$

where $\mu_{i}=\left(1+q_{i}^{2}\right)^{2} / 4 q_{i}^{2} \bar{\gamma}_{i}$.

Note, that the infinite series in (14) and (17) converge rapidly and about ten terms are necessary for an accuracy at the third significant digit.

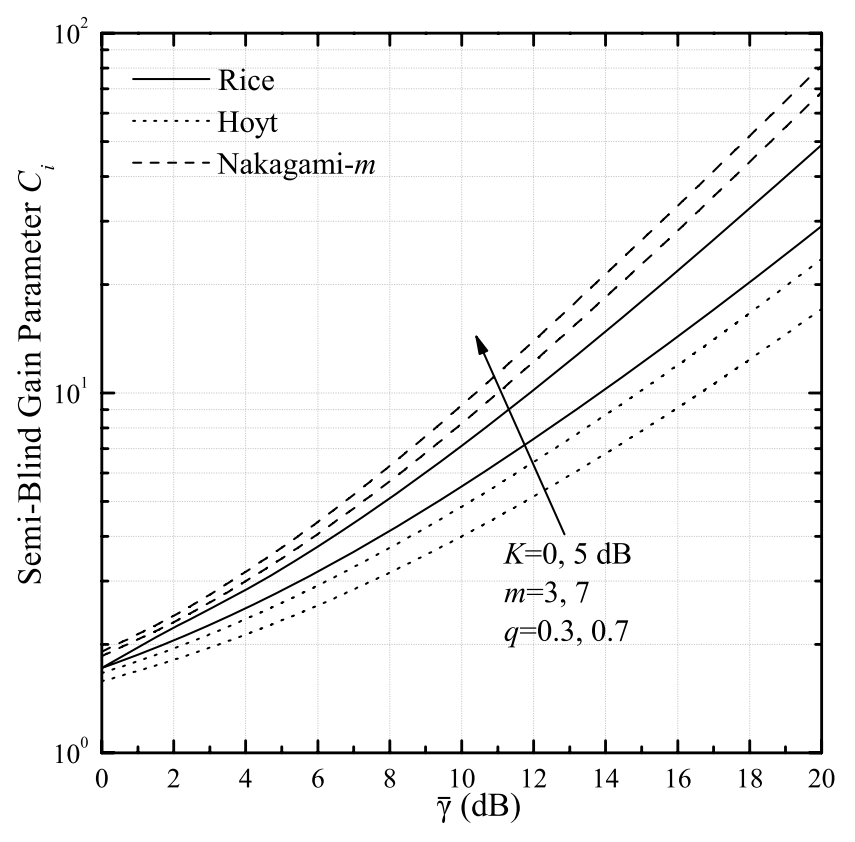

Fig. 1. The semi-blind relay constant $C_{i}$ versus $\bar{\gamma}$ for several values of fading severity parameters $K, q$ and $m$.

3) Nakagami-m fading: For the case of Nakagami- $m$ fading channels the PDF of the fading amplitude $\alpha_{i}$ is given by [11]

$$
f_{\alpha_{i}}\left(\alpha_{i}\right)=\frac{2 \alpha_{i}^{m_{i}}}{\Gamma\left(m_{i}\right) \Omega_{i}^{m_{i}}} \alpha_{i}^{2 m_{i}-1} \exp \left(-\frac{m_{i}}{\Omega_{i}} \alpha_{i}^{2}\right)
$$

with $m_{i}$ being a parameter describing the fading severity of the $i$ th hop. Hence, the gain of the semi-blind relays can be derived in closed-form just averaging (11) over (18) and using [12, eq. (3.353/5) and (8.352/3)] resulting in

$$
G_{i, s b}^{2}=\frac{e^{\lambda_{i}} \lambda_{i}^{m_{i}} \Gamma\left(1-m_{i}, \lambda_{i}\right)}{N_{0, i}}
$$

where $\lambda_{i}=m_{i} / \bar{\gamma}_{i}$. It is easily verified that for $m=1$, (19) reduces to [4, eq. (14)].

In Fig. 1, the semi-blind parameter $C_{i}$ is plotted as a function of the hop's average SNR, assuming for simplification identical hops $\left(\gamma_{1}=\gamma_{2}=\ldots=\gamma_{N}=\bar{\gamma}\right)$, with the same fading parameters $K, q$ and $m$.

\section{Moments of THE END-TO-End SNR}

The first and the second order moments of the end-to-end SNR are statistical parameters which can be efficiently used to evaluate important performance measures, such as average output SNR and variance. The higher order moments (higher than the second one) are also useful in signal processing algorithms for signal detection, classification, and estimation and they play a fundamental role in understanding the performance of wideband communications systems in the presence of fading [13].

Due to the fact that both $\gamma_{e n d}$ and $\gamma_{b}$ are functions of the same positive and statistically independent RVs, $\gamma_{1}, \ldots, \gamma_{N}$, it is easily to prove that

$$
\gamma_{e n d} \leq \gamma_{b} \Rightarrow E\left\langle\gamma_{e n d}^{k}\right\rangle \leq E\left\langle\gamma_{b}^{k}\right\rangle
$$




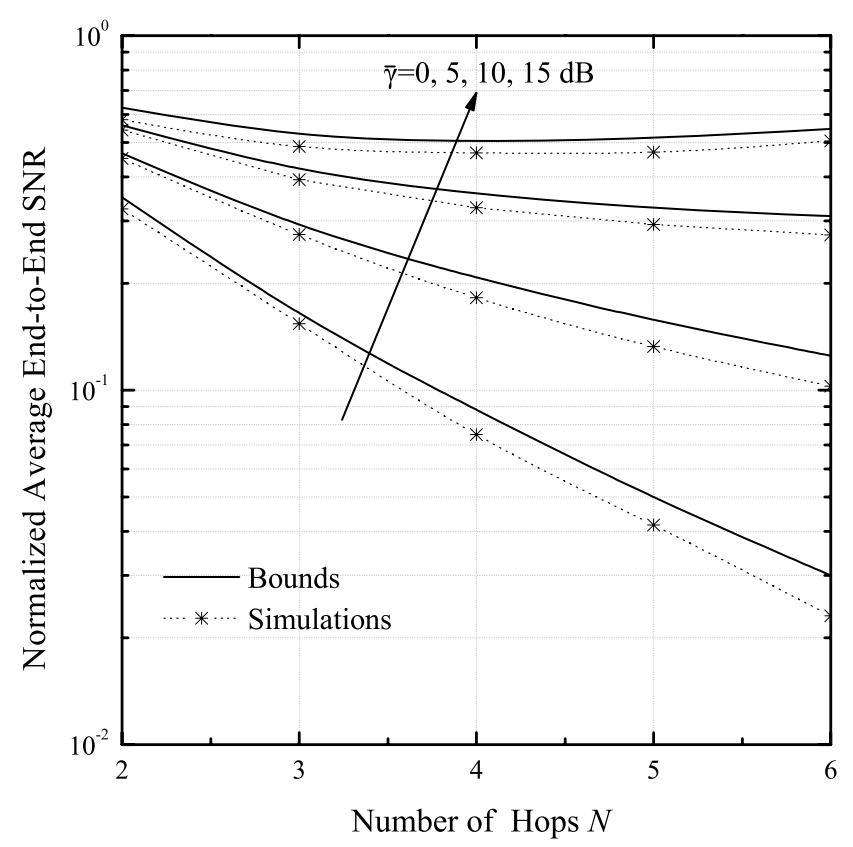

Fig. 2. Normalized average SNR versus the number of hops $N$ in Rician fading with $K=3 \mathrm{~dB}\left(\bar{\gamma}_{i}=\bar{\gamma}\right)$.

Due to the independency of $\gamma_{1}, \gamma_{2}, \ldots, \gamma_{N}$, the $k$ th moment of $\gamma_{e n d}$ can be bounded as

$$
\begin{aligned}
E\left\langle\gamma_{\text {end }}^{k}\right\rangle \leq E\left\langle\gamma_{b}^{k}\right\rangle & =\mathcal{Z}_{N}^{k} E\left\langle\prod_{i=1}^{N} \gamma_{i}^{\frac{k(N+1-i)}{N}}\right\rangle \\
& =\mathcal{Z}_{N}^{k} \prod_{i=1}^{N} E\left\langle\gamma_{i}^{\frac{k(N+1-i)}{N}}\right\rangle
\end{aligned}
$$

\section{A. Nakagami-n (Rice) Fading}

When $\alpha_{i}$ follows Rice distribution, the moments $E\left\langle\gamma_{i}^{k}\right\rangle$ are given by [11, eq. (2.18)]

$$
E\left\langle\gamma_{i}^{k}\right\rangle=\bar{\gamma}_{i}^{k} \frac{\Gamma(1+k)}{\left(1+K_{i}\right)^{k}}{ }_{1} F_{1}\left(-k, 1 ;-K_{i}\right)
$$

with ${ }_{1} F_{1}(\cdot, \cdot ; \cdot)$ is the Kummer confluent hypergeometric function [9, eq. (13.1.10)]. Using (21) and (22) the moments of $\gamma_{b}$ can be expressed in closed-form as

$$
\begin{aligned}
E\left\langle\gamma_{b}^{k}\right\rangle & =\mathcal{Z}_{N}^{k} \prod_{i=1}^{N}\left[\left(\frac{\bar{\gamma}_{i}}{1+K_{i}}\right)^{k(N+1-i) / N}\right. \\
& \times \Gamma\left(1+\frac{k(N+1-i)}{N}\right) \\
& \left.\times{ }_{1} F_{1}\left(-\frac{k(N+1-i)}{N}, 1 ;-K_{i}\right)\right] .
\end{aligned}
$$

In Fig. 2, an upper-bound for the average end-to-end SNR, normalized to the average SNR of the first hop, is plotted versus the number of hops, when Rician fading is considered. Monte-Carlo simulations were also performed and their results are depicted in the same figure showing the accuracy and the tightness of the proposed upper-bound. Moreover, as it was expected, the normalized average end-to-end SNR decreases with the increase of the number of hops. In this figure, it is assumed for simplicity that $\bar{\gamma}_{i}=\bar{\gamma}$ and $K_{i}=K$. Note, that (23) can be efficiently applied also for non-identical hops.

\section{B. Nakagami-q (Hoyt) Fading}

For Nakagami- $q$ (Hoyt) fading channels, $E\left\langle\gamma_{i}^{k}\right\rangle$ can be written using [11, eq. (2.13)] as

$$
E\left\langle\gamma_{i}^{k}\right\rangle=\Gamma(1+k)_{2} F_{1}\left(-\frac{k-1}{2},-\frac{k}{2} ; 1,\left(\frac{1-q_{i}^{2}}{1+q_{i}^{2}}\right)^{2}\right) \bar{\gamma}_{i}^{k}
$$

where ${ }_{2} F_{1}(\cdot, \cdot ; \cdot, \cdot)$ is the Gauss Hypergeometric function [9, eq. (15.1.1)]. Using (21) and (24), $E\left\langle\gamma_{b}^{k}\right\rangle$ can be written in closed-form as

$$
\begin{gathered}
E\left\langle\gamma_{b}^{k}\right\rangle=\mathcal{Z}_{N}^{k} \prod_{i=1}^{N}\left[\bar{\gamma}_{i}^{k(N+1-i) / N} \Gamma\left(1+\frac{k(N+1-i)}{N}\right)\right. \\
\left.\quad \times{ }_{2} F_{1}\left(-\frac{\frac{k(N+1-i)}{N}-1}{2},-\frac{\frac{k(N+1-i)}{N}}{2} ; 1,\left(\frac{1-q_{i}^{2}}{1+q_{i}^{2}}\right)^{2}\right)\right] .
\end{gathered}
$$

\section{Nakagami-m Fading}

For Nakagami- $m$ fading channels, $E\left\langle\gamma_{i}^{k}\right\rangle$ can be written using [11, eq. (2.23)] as

$$
E\left\langle\gamma_{i}^{k}\right\rangle=\bar{\gamma}_{i}^{k} \frac{\Gamma\left(m_{i}+k\right)}{\Gamma\left(m_{i}\right) m_{i}^{k}}
$$

Using (21) and (26), $E\left\langle\gamma_{b}^{k}\right\rangle$ can be derived in closed-form as

$$
E\left\langle\gamma_{b}^{k}\right\rangle=\mathcal{Z}_{N}^{k} \prod_{i=1}^{N}\left[\left(\frac{\bar{\gamma}_{i}}{m_{i}}\right)^{\frac{k(N-i+1)}{N}} \frac{\Gamma\left(m_{i}+\frac{k(N-i+1)}{N}\right)}{\Gamma\left(m_{i}\right)}\right]
$$

and for the Rayleigh fading channels $\left(m_{i}=1\right),(27)$ reduces to

$$
E\left\langle\gamma_{b}^{k}\right\rangle=\mathcal{Z}_{N}^{k} \prod_{i=1}^{N}\left[\bar{\gamma}_{i}^{\frac{k(N-i+1)}{N}} \Gamma\left(1+\frac{k(N-i+1)}{N}\right)\right] .
$$

\section{PERformance Assessment}

\section{A. Average Error Rates}

Using the formulated in (9) upper-bound for the end-to-end SNR and the results of Section III, efficient lower bounds can be derived for the average symbol error probability (ASEP) of several digital modulation schemes by using the momentsbased approach, presented in [8]. According to this method, when the moments of the SNR exist and are expressed in closed-form, the moment generating function (MGF) of $\gamma_{b}$, $\mathcal{M}_{\gamma_{b}}(s)$, can be accurately approximated by using the Padé approximants theory. This, in sequel, allows the evaluation of the average error rates for a wide variety of modulation schemes using the well-known MGF-based approach [11].

Fig. 3 depicts the error performance of BPSK for a multihop system operating in Nakagami- $m$ fading when i.d. and noni.d. mean SNRs per hop are assumed. The accuracy and the tightness of the proposed bound are evident, especially at low SNRs $(<10 \mathrm{~dB})$. Furthermore, it is observed that the bound is more tight, for the i.d. case, due to the nature of the harmonicgeometric mean inequality. Curves for DPSK in Rician fading are also plotted in Fig. 4 while corresponding curves from simulations are plotted only for $N=4$, to avoid entanglement. 


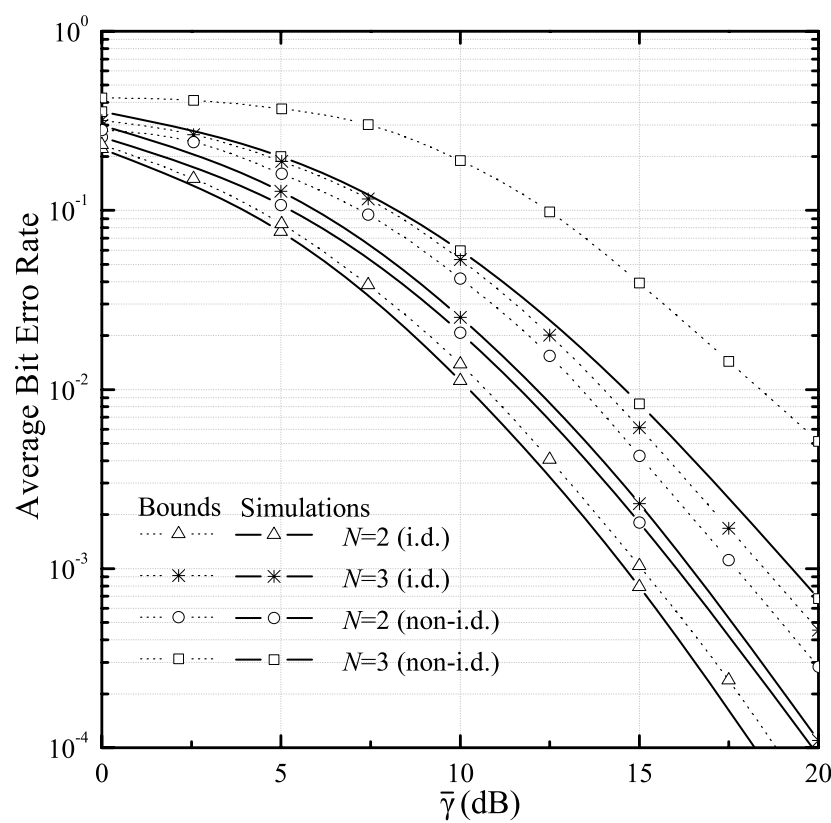

Fig. 3. Error performance for BPSK in Nakagami- $m$ fading $\left(m_{i}=m=3\right.$, i.d.: $\bar{\gamma}_{i}=\bar{\gamma}$, non-i.d.: $\bar{\gamma}_{i}=\bar{\gamma} / i$ ).

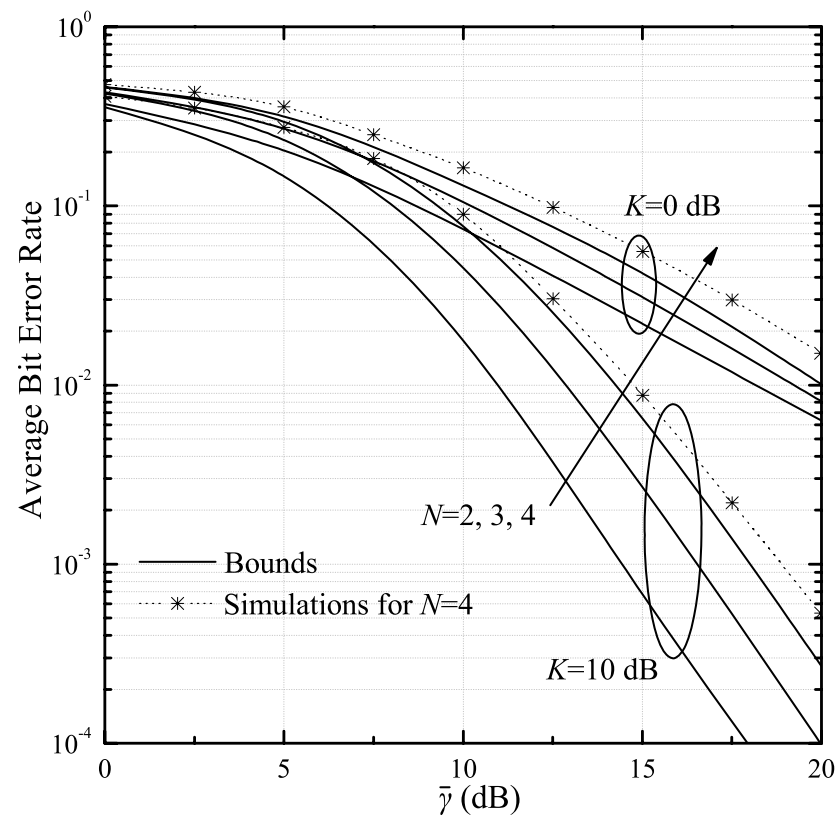

Fig. 4. Error performance for DPSK in Rician fading $\left(K_{i}=K, \bar{\gamma}_{i}=\bar{\gamma}\right)$.

\section{B. Outage Probability}

If $\gamma_{t h}$ is a certain specified threshold ratio, then for nonregenerative multihop transmissions the outage probability is defined as the probability that the instantaneous SNR at terminal D falls below $\gamma_{t h}$. When we use the upper bound $\gamma_{b}$ instead of $\gamma_{e n d}$, then the outage probability is lower bounded, i.e.

$$
P_{\text {out }} \geq F_{\gamma_{b}}\left(\gamma_{t h}\right)
$$

where $F_{\gamma_{b}}\left(\gamma_{t h}\right)$ is the cumulative distribution function of $\gamma_{b}$. If the moments-based approach [8], is used to determine

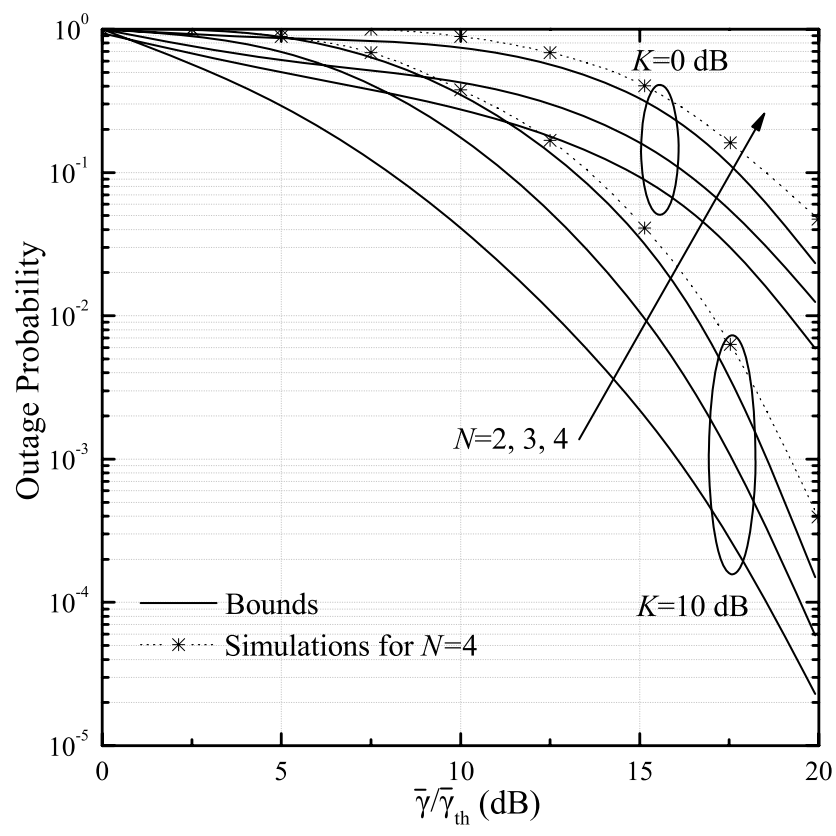

Fig. 5. Outage probability in Rician fading $\left(K_{i}=K, \bar{\gamma}_{i}=\bar{\gamma}\right)$.

$F_{\gamma_{b}}\left(\gamma_{t h}\right)$ from $\mathcal{M}_{\gamma_{b}}(s)$, then

$$
F_{\gamma_{b}}\left(\gamma_{t h}\right)=\sum_{i=1}^{\Phi} \frac{q_{i}}{p_{i}} e^{-p_{i} \gamma_{t h}}
$$

where $p_{i} s$ are the poles of the Padé approximants to $\mathcal{M}_{\gamma_{b}}(s)$, which must have negative real part and $q_{i} s$ are the residues.

In Fig. 5, the outage probability of the multihop system operating in a Rician fading environment is plotted versus the inverse normalized threshold $\bar{\gamma} / \gamma_{t h}$, for several values of $N$ and $K$. Also here is assumed - without loss of generality that $\bar{\gamma}_{i}=\bar{\gamma}$ and $K_{i}=K$.

\section{CONCLUSion}

The end-to-end SNR for multihop wireless communications systems with non-regenerative blind relays was formulated and upper bounded by using the well-known inequality between harmonic and geometric mean of positive RVs. Simple closedform expressions were given for the moments of the end-toend SNR, in Nakagami- $n$ (Rice), Nakagami- $m$ and Nakagami$q$ (Hoyt) generalized fading channels. The outage probability and the average error probability for coherent and noncoherent modulation schemes were studied using the momentbased approach. Furthermore, new formulae were given for the gain of previously proposed semi-blind relays in generalized fading environments. Numerical and computer simulations were shown the accuracy and the tightness of the proposed bounds, especially at low SNRs. Finally, it was shown that the mean SNR per hop unbalancing affects positively the tightness of the proposed bounds.

\section{ACKNOWLEDGMENT}

The author gratefully acknowledges University of Patras Ph.D. candidate T. A. Tsiftsis, who prepared simulations. 
Also, thanks to the anonymous reviewers for their careful reading and critique of the manuscript whose comments and suggestions improved the quality of this paper.

\section{REFERENCES}

[1] M. O. Hasna and M. S. Alouini, "End-to-end performance of transmission systems with relays over Rayleigh fading channels," IEEE Trans. Wireless Commun., vol. 2, no. 6, pp. 1126-1131, Nov. 2003.

[2] — "Harmonic mean and end-to-end performance of transmission systems with relays," IEEE Trans. Commun., vol. 52, no. 1, pp. 130135, Jan. 2004.

[3] - "Outage probability of multihop transmission over Nakagami fading channels," IEEE Commun. Lett., vol. 7, no. 5, pp. 216-218, May 2003.

[4] — , "A performance study of dual-hop transmissions with fixed gain relays," IEEE Trans. Wireless Commun., to appear.

[5] P. A. Anghel and M. Kaveh, "Exact symbol error probability of a cooperative network in a Rayleigh-fading environment," IEEE Trans. Wireless Commun., vol. 3, no. 5, pp. 1416-1421, Sept. 2004.

[6] J. Boyer, D. D. Falconer, and H. Yanikomeroglu, "Multihop diversity in wireless relaying channels," IEEE Trans. Commun., vol. 52, no. 10, pp. 1820-1830, Oct. 2004.
[7] J. N. Laneman, D. N. C. Tse, and G. W. Wornell, "Cooperative diversity in wireless networks efficinet protocols and outage behaviour," IEEE Trans. Inform. Theory, vol. 50, no. 12, pp. 3062-3080, Dec. 2004.

[8] G. K. Karagiannidis, "Moments-based approach to the performance analysis of equal-gain diversity in Nakagami- $m$ fading," IEEE Trans. Commun., vol. 52, no. 5, pp. 685-690, May 2004.

[9] M. Abramovitz and I. A. Stegun, Handbook of Mathematical Functions with Formulas, Graphs, and Mathematical Tables, 9th ed. New York: Dover, 1972.

[10] F. Berggren and S. B. Slimane, "A simple bound on the outage probability with lognormally distributed interferers," IEEE Commun. Lett., vol. 8, no. 5, pp. 271-273, May 2004.

[11] M. K. Simon and M.-S. Alouini, Digital Communication over Fading Channels, 2nd ed. New York: Wiley, 2005.

[12] I. S. Gradshteyn and I. M. Ryzhik, Table of Integrals, Series, and Products, 6th ed. New York: Academic, 2000.

[13] M. Z. Win, R. K. Mallik, and G. Chrisikos, "Higher order statistics of antenna subset diversity," IEEE Trans. Wireless Commun., vol. 2, pp. 871-875, Sept. 2003. 